\title{
Die EU als wehrhafte Demokratie, oder: Warum Brüssel eine Kopenhagen- Kommission braucht
}

Jan-Werner Müller

2013-03-13T17:24:09

Kann es innerhalb der Europäischen Union eine Diktatur geben? Vor ein paar Jahren wäre solch eine Frage noch als interessante Spielerei in der politischen Theorie abgetan worden. Angesichts der jüngsten Entwicklungen in Ungarn und Rumänien ist es plötzlich ernst mit einer Möglichkeit, die in Brüssel nie öffentlich thematisiert wurde. Soll die EU nun als Hüterin der Demokratie agieren und die europäischen Völker sozusagen vor sich selber (oder zumindest vor ihren eigenen Regierungen) schützen? Oder wird Brüssel dadurch zu einem paneuropäischen Polit-Polizisten, welcher Bürgern von Lappland bis Lampedusa das einzig wahre Demokratieverständnis vorschreibt? Die Gefahr eines supranationalen Paternalismus sollte man erstnehmen - aber, wie ich in meinem Band Wo Europa endet zu zeigen versucht habe, ist die EU prinzipiell berechtigt, zum Schutze nationaler Demokratien zu intervenieren. Das eigentliche Problem liegt darin, dass es bis heute keine überzeugenden politischen oder rechtlichen Instrumente für derartige Eingriffe gibt. Einige der Strategien, welche die EU jüngst gegenüber Ungarn und Rumänien angewandt hat, können leicht als opportunistisch erscheinen - auch wenn sie erfolgreich sein sollten.[1]

Ein kurzer Überblick über die existierenden Möglichkeiten: Artikel 7 gilt - ob nun zu Recht oder nicht - als „nukleare Option“ (Barroso), die niemand wahrnehmen will, oder als "große Keule“ (Westerwelle), die niemand schwingen möchte. Die Hemmungen europäischer Regierungen haben sicherlich damit zu tun, dass offizielle Verurteilungen eines Mitgliedslandes - denn das wäre zweifelsohne die öffentliche Wahrnehmung - der ganzen EU-Kultur von Konsens und Kompromiss diametral entgegengesetzt sind. Vor allem treibt die Nationalstaaten die Sorge, dass Artikel 7 irgendwann gegen sie selber angewandt werden könnte. Hier enthüllt sich einmal mehr, welch fatale Folgen der Exekutivföderalismus (Jürgen Habermas), der derzeit in der EU herrscht, haben kann: Nationale Regierungen denken nicht in gesamteuropäischen Zusammenhängen, sondern zeigen sich zuerst einmal als „souveränitätssensibel“ (Mattias Kumm).

Armin von Bogdandy und seine Mitstreiter haben bekanntlich einen ingeniösen Vorschlag zu einem Rettungsschirm für Grundrechte vorgeschlagen, der im Idealfalle auch nationale Gerichte gegenüber illiberalen Kräften stärkt. Aber einmal dahingestellt, ob illiberale Regierungen sich wirklich noch von Urteilen des EuGH beeindrucken lassen würden und ob in Situationen wie in Rumänien im Sommer 2012 die Mühlen der Justiz nicht viel zu langsam mahlen - in den Augen von Kritikern ist der Ansatz zu legalistisch, geht es doch im Kern um politische Herausforderungen. 
Eine direkte politische Antwort aber ist erst einmal nicht zu erwarten. Die Eurokrise, so hört man häufig, habe zur Politisierung Europas geführt - nun gelte es, die Politik zu europäisieren. Doch führt dies offenbar auch dazu, dass sich die Europäische Volkspartei vor Orbán stellt und Martin Schulz, einer der schärfsten Kritiker Orbáns, zumindest am Anfang das Vorgehen des nominell linken rumänischen Ministerpräsidenten Ponta rechtfertigte: Parteipolitik statt Protektion der Demokratie.

Was bräuchte es also? Mit einem Satz gesagt: Eine Institution, die kurzfristig eine Art politischen Alarm auslösen kann - und welche über starke politische Urteilskraft sowie ausreichende Autorität verfügt, damit ein solcher Alarm auch ernst genommen wird. Eine derartige Institution kann einem Gericht in mancher Hinsicht ähneln, aber sie kann keine Kopie eines Gerichtes sein: Sie muss nicht nur individuelle Rechtsverletzungen in den Blick nehmen, sondern die gesamte politische Landschaft erfassen können; ihre Mitglieder sollten ein Gespür haben für Kontexte und Verhältnismäßigkeit. Die Institution muss überparteiisch sein, aber eben nicht im strikten Sinne unpolitisch, weil es ja gerade um dezidiert politische Herausforderungen (und den Blick auf ein politisches Gesamtbild) geht.

Nur: Wird hier nicht das Rad neu erfunden? Hängt der Prozess der EU-Erweiterung nicht von genau dieser Art Urteilen ab? Waren die „Kopenhagen-Kriterien“, welche unter anderem Demokratie und Rechtsstaat forderten, nicht eben jene Art von Kriterien, welche man nun mühsam wieder neu zu formulieren sucht? Es leuchtet ein, dass die vor der großen Erweiterung 2004 gewonnenen Erfahrungen fruchtbar gemacht werden sollten. Es ist aber auch zu bedenken, dass der Erweiterungsprozess eben oft im „Checklist-Verfahren“ vorgenommen wurde: Beamte machten Häkchen bei den einzelnen Kästchen (,Haben die Gerichte funktionstüchtige Computer, ja oder nein?"), ohne sich zu fragen, ob denn beispielsweise der Rechtsstaat bei den Bürgern am Ende wirklich als effektiv gelten kann. Urteilskraft - das heißt eben, dass man nicht einfach Regeln anwenden oder Listen abarbeiten kann.

Wünschenswert ist also eine Institution, welche „holistischer" vorgeht, ohne das Erbe der Kopenhagen-Kriterien zu verleugnen. „Kopenhagen-Kommission“, in Anlehnung an die Venedig-Kommission des Europarates, wäre ein passender Name. Idealerweise würde diese Kommission routinemäßig - vielleicht gar jedes Jahr - Berichte über den Zustand des Rechtsstaats und der Demokratie in allen Mitgliedsländern erstellen. Die Alternative wäre jedoch, von vornherein gewisse Länder gesondert zu behandeln, was nicht zu Unrecht den Verdacht von Vorurteilen (besonders gegen „die Osteuropäer") wecken würde. Die Absicht solcher Berichte - ganz im Sinne der Idee eines „umgekehrten Solange“ - bestünde ja auch nicht darin, Krittelei an jedem Aspekt nationaler Institutionen zu legitimieren oder einen letztlich unpolitischen Traum völlig homogener Rechtsstaatlichkeit zu verwirklichen, sondern in dem Versuch, systematische Probleme möglichst rasch paneuropäisch zur Sprache zu bringen. Im Moment verfolgt niemand - aber auch wirklich niemand - die Entwicklungen in einem Land wie Ungarn, der gleichzeitig die Möglichkeit hat, politischen Alarm und gegebenenfalls Sanktionen auszulösen. Mit anderen Worten: In einer EU ohne gemeinsame europäische Öffentlichkeit und ohne kontinuierliche 
Aufmerksamkeit von Institutionen wie der Kommission, bleibt zu viel dem Zufall überlassen.

Warum aber eigentlich nicht die Kommission, also die Europäische Kommission, damit beauftragen, anstatt schon wieder eine neue (teure) Einrichtung zu schaffen (und neue Akronyme, die kein europäischer Bürger mehr versteht)? Es scheint, dass die Kommission in den kommenden Jahren bewusst politisiert werden wird.

Der Präsident könnte direkt gewählt werden; die Parteien könnten im europäischen Verbund mit einem Spitzenkandidaten antreten, der dann von der Mehrheit im Europaparlament zum Präsidenten der Kommission gewählt würde. Es sei hier dahingestellt, ob eine solche Politisierung die Legitimitätsdefizite der EU ausgleichen kann - plausibel ist, dass eine derartige Kommission sich nicht mehr wie bisher als überparteiliche Hüterin der Verträge inszenieren könnte. Die bereits existierenden Verdachtsmomente - das entsprechende Misstrauen wird von Orbán und seinen Verbündeten kräftig geschürt -, beim supranationalen Schutz von Rechtsstaat gehe es eigentlich nur um Parteipolitik oder paneuropäischen Kulturkampf mit anderen Mitteln, würden noch einmal deutlich verstärkt. Man kann also nicht so ohne Weiteres beides gleichzeitig haben: Demokratieschutz und direkt demokratisch legitimierte Institutionen zum Demokratieschutz, weswegen ja auch beispielsweise in der Bundesrepublik nicht Berlin, sondern Karlsruhe Parteien verbieten kann.

Die Kopenhagen-Kommission soll offiziell warnen können, dass in einem Mitgliedsland Rechtsstaat und Demokratie in Gefahr sind. Sanktionen wären mit einer solchen Warnung erst einmal nicht verbunden; die Hoffnung ist jedoch, dass ein solcher Warnschuss überall im gemeinsamen europäischen Haus zu hören sein wird - und im Idealfall allein die dadurch generierte Aufmerksamkeit eine Regierung zum Umlenken veranlasst. Wenn sie es nicht tut, sollten die „Kopenhagener“ die Europäische Kommission dazu veranlassen können, Fördergelder einzufrieren - eine Idee, welche vergangene Woche auch von vier EU-Außenministern in einem Brief an Barroso ins Spiel gebracht worden ist. Weiterhin wären politische Urteilsbildung und Ausführung der Sanktionen getrennt - wobei das Problematische an solchen Sanktionen zweifelsohne wäre, dass sie alle oder im Falle von Strukturfonds im Zweifel die Ärmsten eines Landes treffen würden, statt spezifisch die Regierung. Was „smart sanctions“ in der EU sein könnten - darüber sollte weiter nachgedacht werden.

Im Übrigen sollten die zuerst diskutierten Instrumente intakt bleiben. Idealerweise würde Artikel 7 allerdings noch um die Möglichkeit erweitert, ein Mitgliedsland ganz aus der Union auszuschließen. Ein solches Szenario ist zwar nicht sehr wahrscheinlich, unmöglich ist es allerdings auch nicht. Derzeit könnte eine Militärdiktatur offiziell in der EU verbleiben - nur eben ohne Stimmrechte im Rat. Eigentlich ein skandalöser Zustand und eine völlige Missachtung der Tatsache, dass eine politische Gemeinschaft entweder umfassende Interventionsmöglichkeiten im Innern oder eine Art „right to expel“ haben muss. Derzeit hat die EU weder das eine noch das andere. Man mag ja, in der Folge von Carl Schmitt oder von Robert Spaemann, die Beschwörung von „europäischen Werten“ für willkürlich oder gar kontraproduktiv halten; aber wenn schon nicht die europäischen Werte „den Raum für unsere europäische res publica“ öffnen, wie Joachim Gauck dies in seiner 
Europa-Rede formulierte, so muss es doch zumindest Prinzipien geben, welche diese Raum klar abstecken und sichern.[2]

Um Missverständnissen vorzubeugen: Der hier gemachte Vorschlag soll nicht auf Vereinheitlichung hinauslaufen. Die üblichen pluralistischen europäischen Praktiken und Prinzipien, um nationalen Besonderheiten Rechnung zu tragen (wie die „margin of appreciation“), werden weiterhin angewandt: Bei Dissens zeigt man sich erst einmal verständnisvoll gegenüber seinen „Peers“, versucht mit einer auf Gegenseitigkeit beruhenden normativen Selbstrelativierung dem anderen entgegen zu kommen, möchte eine gemeinsame Sprache zur Rechtfertigung der eigenen Position finden. Regierungen können einer parteipolitisch befreundeten Exekutive immer noch zureden, wieder Räson anzunehmen. Die Kommission kann auch höflich fragen, ob eine Regierung vielleicht Interesse hätte, Verhandlungen über einen Austritt aus der EU aufzunehmen.

Ein letzter Aspekt darf nicht unerwähnt bleiben: Europäische Institutionen müssen den richtigen Ton treffen: Das Problem ist nicht Ungarn, sondern eine bestimmte Regierung; die Probleme dieses und anderer Länder sind nicht einfach Hirngespinste von Populisten; den EU-Beitritt hat nicht jeder Ungar als Segen empfunden. Europa sollte nicht von oben herab belehren, und wenn es Maßnahmen ergreift - dann immer im Namen gemeinsamer und stets wieder gemeinsam neu zu erringender Prinzipien, anstatt Völker wie Demokratie-Penäler zu behandeln, welche etwas schwer von Begriff sind.

[1] Eine Diskussion der Sanktionen gegenüber Österreich im Jahre 2000 sowie Antworten auf die Frage, ob Berlusconis Italien dem heutigen Ungarn und Rumänien vergleichbar ist, finden sich in Wo Europa endet.

[2] Eine ausführliche Diskussion darüber, was diese Prinzipien sind und warum europäische Vielfalt nicht politically almost anything goes heißt, findet sich in Wo Europa endet.

(cc) BY-NC-ND 